\title{
Arbor
}

\section{Ampliación de la Unión Europea: estado de la cuestión}

\section{Eneko Landaburu}

Arbor CLXXII, 678 (Junio 2002), 345-354 pp.

\section{Introducción: Una ampliación sin precedentes}

La ampliación es el gran desafío de la Unión Europea para los próximos años, el asunto más importante que debe tratarse durante los mandatos de la Comisión Prodi y del actual Parlamento Europeo. ¿Por qué? Porque esta ampliación no se asemeja a ninguna otra ampliación anterior y ninguna volverá a tener el peso real y simbólico de ésta. Se trata de reunir Europa, de reconciliar la geografía con la historia y con la evolución política que la ha transformado en el último decenio, de extender la zona de paz y estabilidad política, de democracia, de respeto de los derechos humanos y de desarrollo económico que representa la Unión Europea. Ninguna ampliación hasta ahora ha tenido que tratar un número tan elevado de nuevas candidaturas: doce países, sin contar Turquía ${ }^{1}$, con una población total de 120 millones de personas. Ninguna ampliación se ha encontrado con países que en el momento de la aceptación de su candidatura, en 1993, tuvieran tan poco en común con las sociedades y las economías de los países miembros de la Unión Europea.

Es una tarea ingente para todas las partes. Para los países candidatos, para quienes la adaptación necesaria para cumplir los criterios políticos y económicos de adhesión y la adopción de las normas comunitarias existentes representan un esfuerzo considerable. Para la Unión, que, por una parte, realiza una inversión importantísima para contribuir a la preparación de los países candidatos y, por otra, debe prepararse por su parte a acoger un gran número de nuevos miembros.

Por consiguiente, ninguna ampliación previa ha conocido una asignación de medios tan importante. Por primera vez, una ampliación se apoya en una estrategia de preadhesión iniciada hace aproximadamente 
diez años, que combina la incorporación de la normativa comunitaria, la apertura progresiva de los mercados y el apoyo financiero. El método de la estrategia de ampliación es a la vez riguroso y ambicioso, y ha demostrado hasta ahora ser el instrumento adecuado para hacer avanzar rápidamente este proyecto histórico.

A continuación se explica cómo se inició este proceso, de qué manera se desarrolla y cómo la Unión, y los países candidatos, lo llevarán a término.

\section{Momentos clave}

\section{Decisiones de base}

El principio de la ampliación de la Unión a nuevos miembros y las condiciones básicas para lograrlo figuran en el Tratado de la Unión Europea, que estipula ${ }^{2}$ que cualquier Estado europeo podrá puede solicitar el ingreso como miembro de la Unión siempre que respete los principios de libertad y democracia, los derechos humanos y las libertades fundamentales. Esta solicitud se debe dirigir al Consejo, que se pronuncia por unanimidad después de haber consultado a la Comisión y previo dictamen conforme del Parlamento Europeo, el cual se pronunciará por mayoría absoluta de los miembros que lo componen.

El Consejo Europeo de Copenhague de 1993 dio un paso decisivo hacia la ampliación actual al acordar que «los países asociados de Europa Central y Oriental que lo deseen podrán convertirse en miembros de la Unión Europea». A partir de ese momento, la cuestión de la ampliación ya no fue «si» sino «cuando» y el Consejo Europeo definió los principios que se han de respetar para conseguirlo. Los candidatos deben cumplir una serie de criterios políticos y económicos, y asumir las obligaciones propias de los Estados miembros de la Unión ${ }^{3}$. El Consejo Europeo de Madrid de 1995 precisó esta obligación señalando que corresponde a los candidatos crear las condiciones para la aplicación efectiva del conjunto de las disposiciones y normas del Derecho comunitario, condición esencial para establecer un marco de confianza mutua entre los Estados miembros actuales y los que aspiran a serlo.

\section{Inicio de las negociaciones}

Una vez establecidas las decisiones básicas, a finales de 1994 el Consejo Europeo de Essen decidió lanzar la estrategia de preadhesión, desti- 
nada a preparar el camino de la futura ampliación. Más tarde, a finales de 1997, se inició en Luxemburgo el proceso de adhesión global, seguido de la primera conferencia europea celebrada el 30 de marzo de 1998 con los 10 países de Europa Central y Oriental, Malta, Chipre y Turquía. En esa línea, se iniciaron las negociaciones de adhesión con seis países que cumplían los criterios políticos y económicos (Estonia, Polonia, la República Checa, Hungría, Eslovenia y Chipre). Dos años más tarde entraban en negociaciones otros seis países (Letonia, Lituania, Eslovaquia, Bulgaria, Rumania y Malta). Como veremos más adelante, también entonces la Unión tomó medidas para garantizar la cobertura financiera de esta importante empresa.

\section{Calendario}

Por último, el Consejo Europeo de Niza de diciembre de 2000 supuso un paso fundamental con el establecimiento del calendario de la ampliación. La cuestión de la ampliación ya no era «cuando» puesto que la Unión se comprometía a celebrar las negociaciones con los países candidatos dispuestos a hacerlo a finales de 2002 y a acogerlos como miembros de pleno derecho en 2004, año de las próximas elecciones del PE.

La cuestión seguía siendo "cómo", y la respuesta también la dio el Consejo de Niza, adoptando decisiones fundamentales encaminadas a iniciar los ajustes institucionales necesarios para acoger a un gran número de nuevos miembros en la Unión, así como a respaldar la nueva estrategia de negociación propuesta por la Comisión con vistas a acelerar el proceso.

\section{Perspectivas}

Un año más tarde los objetivos se mantienen: en 2001 el Consejo Europeo de Gotemburgo (en junio) y después el de Laeken (en diciembre) confirmaban el planteamiento y el método adoptados y, tomando nota de los progresos realizados por los países candidatos, reiteraban el objetivo de la adhesión de diez de ellos en 2004 (la República Checa, la República Eslovaca, Polonia, Hungría, Eslovenia, Estonia, Letonia, Lituania, Chipre, y Malta). Está en marcha un proceso irreversible: la Unión avanza con determinación hacia una de sus más más importantes realizaciones que, mucho más allá de los beneficios económicos y políticos previstos, marcará el curso de la historia de Europa y la vida de sus pueblos. 


\section{Medios para lograr la ampliación}

\section{Preparar la ampliación: la estrategia de preadhesión}

La primera fase de esta estrategia se remonta a los acuerdos europeos celebrados con los diez países de Europa Central y Oriental y a los acuerdos de asociación con Chipre, Malta y Turquía. Los primeros contemplan la creación de una zona de libre comercio, los segundos llegan hasta el establecimiento de una unión aduanera.

A continuación, a raíz de la llegada y la aceptación de las candidaturas, se crearon asociaciones para la adhesión, que indican, con respecto a cada país candidato, las prioridades a corto y medio plazo para el cumplimiento de los criterios políticos y económicos de adhesión. Esto permite la elaboración de un marco de programación financiera de preadhesión de la Unión, de forma que la Comisión establece sus prioridades sobre la base de los progresos constatados.

Por otra parte, cada país candidato establece un programa nacional para la adopción del acervo comunitario en el que figura el calendario de adopción y aplicación de la normativa comunitaria, así como los medios humanos y financieros destinados a lograrlo.

\section{Instrumentos financieros para apoyar la preparación}

La dotación financiera que destina la Unión Europea a la preparación de la ampliación está a la altura de la empresa. La ayuda financiera a la estrategia de preadhesión se duplicó a partir de $2000^{4}$, pasando de 1,5 millardos de euros a 3 millardos de euros, con tres instrumentos, PHARE, SAPARD e ISPA.

PHARE, con un presupuesto de 1,5 millardos de euros, se concentra en las inversiones para la aplicación del acervo y el «Institution Building», es decir, el refuerzo de la capacidad, principalmente administrativa, de los países que deben aplicar el acervo. ISPA (1 millardo de euros) está dirigido a la financiación de grandes proyectos de infraestructura de transportes y medio ambiente, siguiendo el modelo de los fondos de cohesión. SAPARD (0,5 millardos de euros) es un instrumento de ayuda para la adaptación de la agricultura y para el desarrollo rural.

A estos tres fondos hay que añadir las ayudas de importantes instituciones financieras internacionales, en particular el BEI, con el que PHARE e ISPA cofinancian numerosos proyectos de infraestructura. 
Ampliación de la Unión Europea: estado de la cuestión

Chipre, Malta y Turquía no son beneficiarios del programa PHARE; Chipre y Malta reciben apoyo de preadhesión por valor de 95 millones de euros en el marco de un Reglamento financiero único para 2000-2004. El apoyo a Turquía se recoge en un Reglamento que agrupa las distintas líneas presupuestarias disponibles, y su valor anual es de aproximadamente 180 millones de euros.

Hay que señalar que a pesar de haberse duplicado, el apoyo de preadhesión comunitario sólo cubre una pequeña parte de las grandes inversiones necesarias para la preparación de los países candidatos a la adhesión. La financiación pública no es suficiente para cubrir estas necesidades; por consiguiente, la financiación privada debe desempeñar un importante papel, por ejemplo en sectores, como el del medio ambiente, en que puede recurrirse a las concesiones.

\section{Programas de asistencia innovadores: hermanamiento entre administraciones}

La incorporación del acervo comunitario no es un ejercicio de mera transposición. Ajustarse al «tercer criterio» de adhesión supone que los países candidatos han de demostrar su capacidad de asumir compromisos creíbles y de mantenerlos. En otras palabras, aplicar a través de estructuras adecuadas (administrativas, judiciales, de seguridad o de control de la gestión financiera) la compleja y variada normativa de la Unión. Y a este respecto hay que tener en cuenta que el desafío es mayor para los actuales países candidatos de lo que lo fue para los países que se adhirieron con anterioridad. No sólo debido al retraso acumulado bajo los regímenes económicos y políticos existentes hasta hace apenas un decenio, sino también porque, al compás de los progresos de la Unión, el acervo comunitario no ha cesado de enriquecerse durante estos últimos años.

Para apoyar el desarrollo de la capacidad administrativa de estos países («institutions building»), desde 1998 se organiza el desplazamiento de expertos de las Administraciones nacionales de los Estados miembros a las de los países candidatos a través de un programa de hermanamiento. Estos intercambios tienen objetivos precisos, como la instauración de estructuras administrativas modernas y eficaces, con competencias y recursos adecuados para aplicar todos los componentes del acervo, tal como se hace en los Estados miembros. El Consejo Europeo de Laeken aprobó un plan de acción específico, recomendado por la Comisión, para apoyar los esfuerzos de los países candidatos en este ámbito. 
En su calidad de guardiana de los Tratados, la Comisión desempeña un papel extremadamente importante a este respecto; por voluntad de los Estados miembros, ha de efectuar el seguimiento metódico y constante de la observancia de los compromisos asumidos. Los Informes periódicos presentados anualmente por la Comisión tienen por objeto, entre otras cosas, evaluar si se dan las condiciones necesarias para avanzar hacia la adhesión.

\section{Negociar la ampliación: estrategia}

En la actualidad están en curso negociaciones de adhesión con diez países de Europa Central y Oriental, así como con Malta y Chipre. Las negociaciones con Turquía todavía no se han iniciado, debido a que se necesitan avances más importantes para cumplir el criterio político de la adhesión, de manera que los esfuerzos se concentran en la creación de los instrumentos de preadhesión todavía inexistentes.

\section{Principios de la negociación}

Las negociaciones de adhesión se basan en el principio de que los candidatos deben adoptar la totalidad del acervo comunitario. En el marco de las negociaciones, el acervo se ha dividido en 31 capítulos (tales como la agricultura, la competencia o los transportes) denominados «abiertos» o «temporalmente cerrados», dependiendo de que las negociaciones con cada país estén en curso o hayan finalizado ${ }^{5}$. Como el acervo comunitario propiamente dicho no es negociable, se tratan principalmente las condiciones en que se aplica, es decir, si es posible aplazar su adopción y aplicación, mediante medidas transitorias, y en qué condiciones, sin poner en peligro el mercado interior ni la competencia, que son los objetivos que deben suscribir todos los candidatos.

Otro principio subyacente de las negociaciones es el concepto de diferenciación: la rapidez de las negociaciones depende del grado de preparación de cada país candidato y de la complejidad de los problemas que deben solucionarse, examinados individualmente. De ello se desprende que existe la posibilidad de que los países que hayan iniciado más tarde las negociaciones recuperen el atraso, para que cada candidato es juzgado en función de sus propios méritos. 
Ampliación de la Unión Europea: estado de la cuestión

\section{La estrategia de ampliación después de Niza}

El Consejo Europeo de Niza adoptó un elemento suplementario del proceso de negociación: el del «itinerario» o plan de trabajo propuesto por la Comisión para el desarrollo de las negociaciones hasta mediados de 2002. La Unión Europea se compromete a definir en un calendario preciso su posición sobre los «capítulos» (o ámbitos políticos) del acervo comunitario que deben negociarse con cada uno de los países candidatos. Los capítulos se han agrupado en tres series que se tratarán, sucesivamente, durante los tres semestres que van hasta mediados de 2002. En junio y diciembre de 2001, el Consejo Europeo ratificó este método, y el calendario anejo, como marco de la celebración satisfactoria de las negociaciones de adhesión.

\section{Avances rápidos}

Está comprobado que las cosas avanzan: algunos países candidatos ya están muy cerca del objetivo. Si se estudian los «capítulos» del acervo, se ve que en enero se habían abierto 29 capítulos con todos los países en negociación (excepto Bulgaria y Rumania). Algunos capítulos están ya temporalmente cerrados con todos los países, por ejemplo los relativos al derecho de sociedades, las PYME, la ciencia y la investigación, las estadísticas, la educación y la formación, la protección de los consumidores, las relaciones exteriores y la política exterior y de seguridad común. Eslovenia, Polonia, Chipre y la República Checa han «cerrado» más de dos tercios.

Algunos capítulos complejos, como la libre circulación de bienes y personas, la industria y la pesca, están prácticamente concluidos con casi todos los candidatos. Por otro lado, algunos capítulos todavía no se han cerrado con ningún país, por ejemplo la agricultura, la política regional y los presupuestos. Por supuesto, esto no significa que no se haya conseguido nada: entretanto se han llevado a cabo todos los trabajos preparatorios a fin de solucionar los apartados menos problemáticos e identificar las cuestiones más complejas. La Comisión, que deberá pronunciarse sobre estos capítulos antes de finales de año, presentó a los Estados miembros a finales de noviembre propuestas sobre la política regional y a finales de enero un documento sobre el capítulo agrícola, que contempla los mecanismos de gestión.

Para los capítulos aún abiertos, las negociaciones continúan de acuerdo con el calendario fijado por el «itinerario» en el marco de la estrategia 


\section{Eneko Landaburu}

de ampliación. En particular, para los países con los que las negociaciones se han abierto más recientemente, el método de apertura de los capítulos se basa en el estado de su preparación por parte de los países en cuestión y en la capacidad de estos últimos de presentar propuestas de negociación. A partir de ese momento, se registra un movimiento de aceleración constante del proceso.

\section{Cuestiones abiertas}

\section{Adaptaciones institucionales: el Convenio sobre el futuro de la Unión}

Con la celebración de la Conferencia Intergubernamental sobre la reforma de las instituciones, en Niza se suprimió el obstáculo institucional a la ampliación. Las características de las instituciones de la Unión ampliada están ahora claras (número de Comisarios, de Parlamentarios europeos, de votos en el Consejo para cada uno de los candidatos con los cuales se negocia). El Consejo Europeo de Laeken de diciembre pasado presentó el Convenio sobre el Futuro de Europa, para definir los límites y el funcionamiento de la Unión de mañana, en el horizonte de 2004. Los países candidatos participan en este ejercicio, ya que está claro que la Unión de mañana será sobre todo una Unión más extensa.

\section{Abrir la cuestión a los ciudadanos}

La próxima fase tras las negociaciones será la celebración de los Tratados de adhesión y más tarde su ratificación. Será un momento crucial, puesto que es cuando los esfuerzos realizados por los países candidatos y la Unión para preparar cuidadosamente esta etapa histórica serán sancionados por sus habitantes.

Sin excluir la celebración de consultas populares en los países miembros, están previstos referéndums en varios países candidatos. Si bien la ampliación aportará ventajas políticas, geopolíticas y socioeconómicas a todas las partes, y aunque estas ventajas ya son perceptibles en términos de empleo, de ampliación del mercado y de aumento de los intercambios, hay que reconocer que por ahora las opiniones públicas no están muy impresionadas.

Seguramente es porque el proceso, sus implicaciones, lo que está en juego y su cuidadosa preparación no se comprenden bien. Sin duda se debe a 
que esta fase la preparación de la ampliación es el coto de los especialistas, los expertos y los inspectores, ya que los aspectos técnicos no son fáciles de comunicar. Al menos, se debería poder calmar los temores expresados en algunos sectores, a los que habría que recordar que la negociación de la ampliación tiene en cuenta los intereses de cada una de las partes.

Pero no debemos contentarnos con esta constatación ya que en última instancia, la ampliación sólo podrá hacerse si los ciudadanos la desean. Y ello sólo será posible si la ampliación deja de ser un asunto exclusivo del Estado y de los responsables políticos. El debate no debe seguir confinado a un estrecho círculo de interesados sin que la sociedad civil y los ciudadanos estén informados del increíble esfuerzo de preparación, de la amplitud de las reformas emprendidas por los países candidatos, de la inversión realizada y, sobre todo, de los cambios que se perfilan en un horizonte ya muy cercano. La Comisión Europea y las demás instituciones, en particular el Parlamento Europeo, han tomado conciencia de este deber de información y elaboran actualmente una estrategia de comunicación para explicar lo que hay que explicar en los países miembros y los países candidatos. Ahora bien, la información sólo llega si se transmite y se discute; a este respecto, la responsabilidad de los que «saben» es hablar a los que escuchan.

Asimismo, es necesario ampliar el contenido del debate, que debe superar las consideraciones técnicas y la presentación de las ventajas macroeconómicas y políticas a medio y largo plazo para concentrarse más en las cuestiones importantes para los ciudadanos.

Reducir la ampliación a su preparación política, financiera, técnica o incluso a las ventajas individuales que acarreará, sería reductor, ya que la ampliación de la Unión no es un cálculo de gestores, ni una apuesta por el futuro político de nuestro continente.

La ampliación es un proyecto para el futuro de Europa, para la vida de todos los ciudadanos de los Estados miembros actuales y de los de mañana. Ciudadanos que tienen en común Europa, una comunidad de patrimonio histórico, cultural y geográfico enriquecida por la diversidad de los matices que la constituyen. Ampliar la Unión es crear un nuevo espacio para que los hombres y las mujeres, nuestros conciudadanos, se la apropien, para que haya un intercambio recíproco, se conozcan y crezcan. Es recrear Europa para, unidos, hacerla revivir.

\section{Notas}

Información complementaria y actualizaciones diarias disponibles en el sitiola página web siguiente: 
1 Los países candidatos son 13: Bulgaria, Chipre, la República Checa, la República Eslovaca, Eslovenia, Estonia, Letonia, Lituania, Malta, Polonia, Hungría, Rumania y Turquía. Las negociaciones están en curso con 12 de ellos; Turquía, en efecto, no cumple aún el primer criterio de adhesión (el criterio político: la existencia de un Estado democrático, de derecho, que garantice el respeto de los derechos humanos y la protección de las minorías).

${ }^{2}$ Artículo 49 del Tratado de la Unión Europea, modificado por el Tratado de Amsterdam.

${ }^{3}$ Los criterios de adhesión, frecuentemente denominados "criterios de Copenhague", son los siguientes:

- la existencia de instituciones estables que garanticen la democracia, el Estado de Derecho, el respeto de los derechos humanos y la protección de las minorías («criterio político");

- la existencia de una economía de mercado en funcionamiento, así como la capacidad para hacer frente a la presión competitiva y a las fuerzas del mercado dentro de la Unión («criterio económico»);

- la capacidad de asumir las obligaciones de la adhesión, incluida la observancia de los fines de la Unión Política, Económica y Monetaria («tercer criterio»).

4 En marzo de 1999, el Consejo Europeo de Berlín definió el marco financiero de la estrategia de preadhesión para el período 2000-2006 ("Agenda 2000 »). Cabe señalar que el Consejo Europeo de Laeken de finales de 2001 confirmó estas perspectivas financieras por corresponder a las necesidades de la ampliación a 10 nuevos miembros.

5 Los capítulos se considerarán definitivamente "cerrados" cuando se cierren todos los capítulos para un país dado. 